\title{
Estimate of an Hypoelliptic Heat-Kernel outside the Cut-Locus in Semi-Group Theory
}

\author{
Rémi Léandre \\ Laboratoire de Mathematiques, Université de Franche-Comté, Besançon, France \\ Email: remi.leandre@univ-fcomte.fr
}

Received July 2, 2012; revised August 2, 2012; accepted August 9, 2012

\begin{abstract}
We give a proof in semi-group theory based on the Malliavin Calculus of Bismut type in semi-group theory and Wentzel-Freidlin estimates in semi-group of our result giving an expansion of an hypoelliptic heat-kernel outside the cut-locus where Bismut's non-degeneray condition plays a preominent role.
\end{abstract}

Keywords: Subriemannian Geometry; Heat-Kernels

\section{Introduction}

Let us consider some vector fields $X_{i}, i=1, \cdots, m$ on $\mathbb{R}^{\mathrm{d}}$ with bounded derivatives at each order. We consider the generator

$$
L=1 / 2 \sum X_{i}^{2}
$$

It generates a Markov semi-group $P_{t}$ acting on bounded continuous $f$ functions on $\mathbb{R}^{\mathrm{d}}$. The natural question is to know if the semi-group has an heat-kernel:

$$
P_{t}[f](x)=\int_{\mathbb{R}^{\mathrm{d}}} p_{t}(x, y) f(y) \mathrm{d} y
$$

Let us suppose that the strong Hoermander hypothesis is checked: in such a case Hoermander ([1]) proved the existence of a smooth heat kernel. Malliavin [2] proved again this theorem by using a probabilistic representation of it. A lot of tools of stochastic analysis were translated recently by Léandre in semi-group theory. We refer to the review papers [3]. In particular [4] proved again the existence of the heat kernel by using the Malliavin Calculus of Bismut type in semi-group theory.

Let us recall what is strong Hoermander hypothesis.

Let

$$
\begin{aligned}
E_{1} & =\left\{X_{1}, \cdots, X_{m}\right\} \\
E_{l+1} & =\bigcup_{Y \in E_{l}, i=1, \cdots, m}\left[Y, X_{i}\right]
\end{aligned}
$$

Strong Hoermander hypothesis in $x$ is the following: there exits an $l$ such that

$$
\inf _{|\xi|=1} \sum_{E_{l}}(Y(x), \xi)^{2} \geq C>0
$$

Under Hoermander hypothesis in $x, p_{t}(x, y)$ exists and is smooth in $y$.
Let $h$ be a path from $[0,1]$ into $\mathbb{R}^{m}$ with finite energy

$$
\|h\|^{2}=\int_{0}^{1} \sum_{i=1}^{m}\left|\left(h_{s}^{i}\right)^{\prime}\right|^{2} \mathrm{~d} s<\infty
$$

The Hilbert space of $h$ such that (6) is satisfied is denoted by $\mathbb{H}$.

We consider the horizontal curve $x_{s}(h)$ starting from $x$ :

$$
\mathrm{d} x_{s}(h)=\sum X_{i}\left(x_{s}(h)\right) \mathrm{d} h_{s}^{i}
$$

We consider the control distance $\mathrm{d}(x, y)$

$$
\mathrm{d}^{2}(x, y)=\inf _{x_{0}(x)=x, x_{1}(h)=y}\|h\|^{2}
$$

By standard result of semi-riemannian geometry ([5], [6]), if the Hoermander hypothesis is checked in all $x,(x, y) \rightarrow \mathrm{d}(x, y)$ is finite continuous.

Bismut in his seminal book [7] has introduced the notion of cut-locus associated to the sub-riemannian distance $\mathrm{d}(\cdot, \cdot)$. We will recall in the first part what is the cut-locus in sub-riemannian geometry.

Bismut in his seminal book [3] pointed out the relationship between the Malliavin Calculus, Wentzel-Freidlin estimates and short time asymptotics of heat-kernels. This relationship was fully performed by Léandre in $[8,9]$. In particular, by using probabilistic technics we proved:

Theorem 1. (Léandre [9]). If $x$ and $y$ are not in the cut-locus of the sub-riemannian distance, we have when $t \rightarrow 0$

$$
p_{t}(x, y) \sim C(x, y) t^{-\mathrm{d} / 2} \exp \left[-\mathrm{d}^{2}(x, y) / 2 t\right]
$$

where $C(x, y)>0$. 
In the proof we used a mixture between large deviation estimates, the Malliavin Calculus and the Bismutian procedure. Several authors laters $([10,11])$ have presented other probabilistic proofs of (9). See [12] in a special case. We refer to [13] for an analytic proof of this result.

Remark. The complement of the cut-locus is an opensubset of $\mathbb{R}^{d} \times \mathbb{R}^{d}$ : estimate (9) is uniform on any compact set of the complement of the cut-locus.

For readers interested by short time asymptotics of heat-kernels by using probabilistic methods, we refer to the review papers [14-16] and to the book of Baudoin [17]. We refer to the books of Davies [18] and of Varopoulos-Coulhon-Saloff-Coste [19] for analytical methods and to the review of Jerison-Sanchez [20] and Kupka [6].

The object of this paper is to translate in semi-group theory the proof of Theorem 1 of Takanobu-Watanabe [11], by using the tools of stochastic analysis for estimate of heat kernels we have translated in semi-group theory in $[21,22]$ and $[23]$ for Varadhan type estimates.

\section{The Cut Locus Associated to a Sub-Riemannian Distance}

The material of this part is taken on [7]. But we refer to [11] for a nice introduction to it.

We consider the map $h \rightarrow x_{1}(h)$ starting from $x$. This map is a Frechet smooth function from $\mathbb{H}$ into $\mathbb{R}^{\mathrm{d}}$. We consider $U_{t}=D_{x} x_{t}(h)$. It satisfied the linear equation starting from $I$ :

$$
\mathrm{d} U_{t}=\sum_{i} D_{x} X_{i}\left(x_{t}(h)\right) U_{t} \mathrm{~d} h_{t}^{i}
$$

We get

$$
D x_{1}(h) \cdot k=\sum_{i} U_{1} \int_{0}^{1} U_{s}^{-1} X_{i}\left(x_{s}(h)\right) \mathrm{d} k_{s}^{i}
$$

The Gram matrix associate to the map $h \rightarrow x_{1}(h)$ is

$$
\begin{aligned}
& \int_{0}^{1}\left\langle U_{1} U_{s}^{-1} X_{i}\left(x_{s}(h)\right) \cdot,\right\rangle^{2} \mathrm{~d} s \\
= & \left\langle D x_{1}(h), D x_{1}(h)\right\rangle
\end{aligned}
$$

Bismut introduced the question to know if $h \rightarrow x_{1}(h)$ is a submersion. It is fullfilled if and only if the Gram matrix $\left\langle D x_{1}(h), D x_{1}(h)\right\rangle$ is invertible.

By standard result on Carnot-Caratheodory distance $\mathrm{d}^{2}(x, y)=\|h\|^{2}$ for some $h \in \mathbb{H}$ such that $x_{0}(h)=x, x_{1}(h)=y$.

Let be $K_{x, y}$ the set of $h$ such that $x_{0}(h)=x, x_{1}(h)=y$. The main remark of Bismut [7] is the following: if $h \in K_{x, y}$ and $\left\langle D x_{1}(h), D x_{1}(h)\right\rangle$ is invertible, then $K_{x, y}$ is in a neighborhood of $h$ a submanifold of $\mathbb{H}$ by using the implicit function theorem.
We recall the following definition:

Definition 2. (Bismut [7]) We say that $(x, y)$ are not in the cut-locus of the cut-locus of the sub-riemannian distance $\mathrm{d}(\cdot, \cdot)$ if the following 3 conditions are checked:

1) $\mathrm{d}^{2}(x, y),=\left\|h_{x, y}\right\|^{2}$ for only one element of $K_{x, y}$.

2) The Gram matrix $\left\langle D x_{1}\left(h_{x, y}\right), D x_{1}\left(h_{x, y}\right)\right\rangle$ is invertible.

3) $d^{2}(x, y)$ is a non-degenerated minimum of the energy function $h \rightarrow\|h\|^{2}$ on $K_{x, y}$.

Condition 3) has a meaning because $K_{x, y}$ is a manifold on a neighborhood of $h_{x, y}$.

As traditional in sub-riemannian geometry, we consider the Hamiltonian $H(x, p)$. It is the function from $\mathbb{R}^{\mathrm{d}} \times \mathbb{R}^{\mathrm{d}}$ into $\mathbb{R}^{+}$

$$
(x, p) \rightarrow 1 / 2 \sum\left\langle X_{i}(x), p\right\rangle^{2}
$$

When there is an Hamiltonian, people introduced classically the Hamilton-Jacobi equation associated. In sub-riemannian geometry, this was introduced by Gaveau [24]. A bicharacteristic is the solution of the ordinary differential equation on $\mathbb{R}^{\mathrm{d}} \times \mathbb{R}^{\mathrm{d}}$ starting from $(x, p)$ :

$$
\begin{aligned}
& x_{t}^{\prime}(p)=D_{p} H\left(x_{t}(p), p_{t}(p)\right) \\
& p_{t}^{\prime}(p)=-D_{x} H\left(x_{t}(p), p_{t}(p)\right)
\end{aligned}
$$

We put

$$
h_{t}^{i}(p)=\left\langle X_{i}\left(x_{t}(p)\right), p_{t}(p)\right\rangle
$$

We recall some classical result on sub-riemannian geometry (See [11], p. 204):

$$
\begin{aligned}
& H\left(x_{t}(p), p_{t}(p)\right)=H(x, p) \\
& =1 / 2\|h(p)\|^{2} \\
& p_{t}(p)=\left({ }^{t} U_{t}(h(p))\right)^{-1} p \\
& h(p)=D x_{1}(h(p))^{*} p_{1}(p)
\end{aligned}
$$

Let us recall one of the main result of [7]. If $(x, y)$ does not belong to the cut locus of $d(\cdot, \cdot)$, then $x_{t}\left(h_{x, y}\right)=x_{t}\left(p_{x, y}\right)$ for a convenient bicharectiristic.

By using result of [11] pp. 206-207, we can compute the Hessian of the energy in $h_{x, y}$ in $K_{x, y}$. It is equal to

$$
\begin{aligned}
I^{\prime \prime}(k, l)= & \langle k, l\rangle_{\mathbb{H}} \\
& -\left\langle p_{1}\left(p_{x, y}\right), D^{2} x_{1}\left(h_{x, y}\right) k, l\right\rangle
\end{aligned}
$$

We can compute $D^{2} x_{1}(h)(k, l)$. It is given by 


$$
\begin{aligned}
& D^{2} x_{1}(j)(k, l) \\
& =U_{1}(h)\left\{\sum_{i} \int_{0}^{1} U_{s}(h)^{-1} D_{x}^{2} X_{i}\left(x_{s}(h)\right) D x_{s}(h)(k)\right. \\
& \otimes D x_{s}(h)(l) \mathrm{d} h_{s}^{i} \\
& +\sum_{I} \int_{0}^{1} U_{s}(h)^{-1} D_{x} X_{i}\left(x_{s}(h)\right) \\
& \left.\cdot\left(D x_{s}(h)(k) \mathrm{d} l_{s}^{i}+D x_{s}(h)(l) \mathrm{d} k_{s}^{i}\right)\right\} \\
& =A_{1}(k, l)+A_{2}(k, l)
\end{aligned}
$$

\section{Scheme of the Proof of Theorem 1}

We translate in semi-group the proof of [9] in the way presented in [12].

See [22] for similar considerations for logarithmic estimates of the heat-kernel.

We consider $t=\epsilon^{2}$ classically and introduce the operator

\section{Classically}

$$
L_{\epsilon}=1 / 2 \epsilon^{2} \sum X_{i}^{2}
$$

$$
\exp \left[L_{\epsilon}\right]=\exp [t L]
$$

We consider the unique curve of minimum enegy $h_{x, y}$ sucht $x_{1}\left(h_{x, y}\right)=y$ and we introduce the operator

$$
L_{\epsilon}\left(h_{x, y}\right)=L_{\epsilon}+\sum \mathrm{d} / \mathrm{d} s h_{x, y, s}^{i} X_{i}
$$

This generates a time inhomogeneous semi-group. According the Girsanov formula in semi-group theory of Léandre [4], we introduce the vector field on $\mathbb{R}^{\mathrm{d}} \times \mathbb{R}$ :

$$
\tilde{X}_{i}(\epsilon)=\left(\epsilon X_{i},-1 / \epsilon \mathrm{d} / \mathrm{d} s h_{x, y, s}^{i} u\right)
$$

and the generator written in Itô form

$$
\begin{aligned}
\tilde{L}_{\epsilon}\left(h_{x, y}\right) \tilde{f} & =\sum_{i} \mathrm{~d} / \mathrm{d} s h_{x, y, s}^{i}\left\langle X_{i}, \tilde{D} \tilde{f}\right\rangle \\
& +1 / 2 \epsilon^{2} \sum_{i>0}\left\langle D X_{i} X_{i}, \tilde{D} \tilde{f}\right\rangle \\
& +1 / 2 \sum_{i}\left\langle\tilde{X}(\epsilon), \tilde{D}^{2} \tilde{f}, \tilde{X}_{i}(\epsilon)\right\rangle
\end{aligned}
$$

According [21], p. 207, we have:

$$
\exp \left[L_{\varepsilon}\right][f](x)=\exp \left[\tilde{L}_{\in}\left(h_{x, y}\right)\right][u f](x, 1)
$$

We consider the generator

$$
\bar{L}\left(h_{x, y}\right)=\sum \mathrm{d} / \mathrm{d} s h_{x, y, s}^{i} X_{i}+1 / 2 \sum \tilde{X}_{i}^{2}(\epsilon)
$$

It differs from $\tilde{L}_{\epsilon}\left(h_{x, y}\right)$ by $-1 / 2 \sum\left|h_{x, y, s}^{i}\right|^{2} u D_{u}$. This last vector field commute with $\tilde{L}_{\epsilon}\left(h_{x, y}\right)$. We deduce that

$$
\begin{aligned}
& \exp \left[\tilde{L}_{\epsilon}\left(h_{x, y}\right)\right][u f](x, 1) \\
= & \exp \left[-\mathrm{d}^{2}(x, y) / 2 t\right] \exp \left[\bar{L}_{\epsilon}\left(h_{x, y}\right)\right][u f](x, 1)
\end{aligned}
$$

We consider the vector fields

$$
\bar{Y}_{i}(\epsilon)=\left(\epsilon X_{i},-\mathrm{d} / \mathrm{d} s h_{t}^{i}\right)
$$

and the generator

$$
\bar{Q}_{\epsilon}\left(h_{x, y}\right)=\sum \mathrm{d} / \mathrm{d} s h_{x, y, s}^{i} X_{i}+1 / 2 \sum \bar{Y}_{i}^{2}(\epsilon)
$$

We have clearly that

$$
\begin{aligned}
& \exp \left[\bar{L}_{\epsilon}\left(h_{x, y}\right)\right][u f](x, 1) \\
= & \exp \left[\bar{Q}_{\epsilon}\left(h_{x, y}\right)\right][\exp [u / \epsilon] f](x, 0)
\end{aligned}
$$

Let us consider the flow $\Phi_{s}$ associated to the ordinary differential Equation (7) $x_{s}\left(h_{x, y}\right)$. Let us introduce the vector fields

$$
Y_{i}(\epsilon)=\left(\epsilon \Phi_{s}^{*-1} X_{i},-\mathrm{d} / \mathrm{d} s h_{x, y, s}^{i}\right)
$$

and the time-dependent generator

$$
Q_{\epsilon}\left(h_{x, y}\right)=1 / 2 \sum Y_{i}^{2}(\epsilon)
$$

We have the main formula

$$
\begin{aligned}
& \exp \left[\bar{Q}_{\epsilon}\left(h_{x, y}\right)\right][\exp [u / \epsilon] f](x, 0) \\
= & \exp \left[Q_{\epsilon}\left(h_{x, y}\right)\right]\left[\exp [u / \epsilon] f_{1}\right](x, 0)
\end{aligned}
$$

where $f_{1}$ is the map which to $z$ associate $f\left(\Phi_{1}(z)\right)$. Since $\Phi_{1}(x)=y$, we have only to estimate the density in $x$ of the measure which to $f$ associates

$$
\exp \left[Q_{\epsilon}\left(h_{x, y}\right)\right][\exp [u / \epsilon] f](x, 0)
$$

We can suppose without any restriction that $x=0$.

We perform the dilation $y \rightarrow y / \epsilon$.

This means that we have to consider the vector fields

$$
Z_{i}(\epsilon)=\left(\Phi_{s}^{*-1} X_{i}(\epsilon \cdot),-\mathrm{d} / \mathrm{d} s h_{x, y, s}^{i}\right)
$$

and the generator

$$
R_{\epsilon}\left(h_{x, y}\right)=1 / 2 \sum Z_{i}^{2}(\epsilon)
$$

We consider the density $r_{\varepsilon}(\cdot)$ ot the measure which to the test function $f$ associates

$$
\exp \left[R_{\epsilon}\left(h_{x, y}\right)\right][\exp [u / \epsilon] f](0,0)
$$

The main result of [21] is the following: for some $C(x, y)>0$

$$
\left\{C(x, y) \exp \left[-\mathrm{d}^{2}(x, y) / 2 t\right] / t^{\mathrm{d} / 2}\right\} r_{\epsilon}(0)=p_{t}(x, y)
$$

The main difference with [21] is in treatment of the term $\exp [u / \epsilon]$. We refer to $[9,10,12]$ for the treatment of that expression by using stochastic analysis. 
In Part 2, $D x_{s}\left(h_{x, y}\right) \cdot k$ and $D^{2} x_{s}\left(h_{x, y}\right) k \cdot k$ satisfy a system of stochastic differential equations in cascade with associated vector fields $Y_{i}(1), Y_{i}(2)$. We denote $\left(x^{\prime}, u_{1}, u_{2}\right)$ the generic element of $\mathbb{R}^{\mathrm{d}} \times \mathbb{R}^{\mathrm{d}} \times \mathbb{R}^{\mathrm{d}}$. We consider the vector fields

$$
\bar{Z}_{i}(\epsilon)=\left(\Phi_{s}^{*-1} X_{i}(\epsilon \cdot), Y_{i}(1), Y_{i}(2)\right)
$$

and the generator

$$
\bar{R}\left(h_{x, y}\right)=1 / 2 \sum \bar{Z}_{i}^{2}(\epsilon)
$$

From (14), (15), (18), the density $r_{\epsilon}(0)$ is equal to the density $\bar{r}(0)$ in 0 of the measure which to $f$ associates

$$
\begin{aligned}
& \exp \left[\bar{R}\left(h_{x, y}\right)\right] \\
& \cdot \exp \left[\left\langle\frac{\Phi_{1}\left(\epsilon x^{\prime}\right)-y-\epsilon u_{1}-\epsilon^{2} \frac{1}{2} u_{2}}{\epsilon^{2}}, p_{1}(x, y)\right\rangle\right] \\
& \left.\cdot \exp \left[\left\langle\frac{1}{2} u_{2}, p_{1}(x, y)\right\rangle\right] f\right](0,0,0)
\end{aligned}
$$

where $p_{s}(x, y)$ is associated to $h_{s}(x, y)$ by the procedure of the Part 2. Theorem 1 will follow from Theorem 6.

We consider $\left(x^{\prime}, u_{1}, u_{2}, v\right)$ the generic element of $\mathbb{R}^{\mathrm{d}} \times \mathbb{R}^{\mathrm{d}} \times \mathbb{R}^{\mathrm{d}} \times \mathbb{R}$ and

$$
\bar{Z}_{0}(\epsilon)=\left(0,0,0, \epsilon^{2}\left(x^{\prime}\right)^{2}\right)
$$

and the generator

$$
\tilde{R}_{\epsilon}\left(h_{x, y}\right)=1 / 2 \sum_{i>0} \bar{Z}_{i}^{2}(\epsilon)+\bar{Z}_{0}(\epsilon)
$$

The following lemma is proved in the appendix and was originally proved by stochastic analysis in [12].

Lemma 3. For any positive $p$, there exists a $\rho$ such that

$$
\begin{aligned}
& \exp \left[\tilde{R}\left(h_{x, y}\right)\right] \\
& .\left[\left(\exp \left[\left\langle\frac{\Phi_{1}\left(\epsilon x^{\prime}\right)-y-\epsilon u_{1}-\epsilon^{2} \frac{1}{2} u_{2}}{\epsilon^{2}}, p_{1}(x, y)\right\rangle-1\right)^{p}\right.\right. \\
& \left.\cdot 1_{\left|x^{\prime}\right| \leq \rho} 1_{|| \mid \leq \rho}\right](0,0,0,0,0) \rightarrow 0
\end{aligned}
$$

when $\epsilon \rightarrow 0$

The next lemma is due to Bismut [7] and is proved without using stochastic analysis in the appendix:

Lemma 4. Let $\rho>0$ be very small. There exists a $p>1$ such that

$$
\begin{aligned}
& \exp \left[\tilde{R}_{\epsilon}\left(h_{x, y}\right)\right] \\
& {\left[\left[\exp p\left\langle u_{2}, p_{1}(x, y)\right\rangle / 2\right] 1_{\left|x^{\prime}\right| \leq \rho} 1_{|v| \leq \rho}\right](0,0,0,0)<\infty}
\end{aligned}
$$

The remaining part of the scheme of the proof is to apply the Malliavin Calculus of Bismut type depending of a parameter of [21], Part 3 to the the semi-group $\exp \left[\tilde{R}_{\epsilon}\left(h_{x, y}\right)\right]$. We will apply an improvement of Theorem 1 of [21]. We consider

$$
\left(x^{\prime}, u_{1}, u_{2}, v, U, V\right) \in \mathbb{R}_{\mathrm{d}}=\mathbb{R}^{\mathrm{d}} \times \mathbb{R}^{\mathrm{d}} \times \mathbb{R}^{\mathrm{d}} \times \mathbb{R} \times \mathbb{G}_{\mathrm{d}} \times \mathbb{M}_{\mathrm{d}}
$$

where $\mathbb{G}_{\mathrm{d}}$ is the set on invertible matrices on $\mathbb{R}^{\mathrm{d}}$ and $\mathbb{M}_{d}$ the set of symmetric matrices on $\mathbb{R}^{\mathrm{d}}$ ( $V$ is called the Malliavin matrix). We consider if $i>0$ the vector fields on $\mathbb{E}_{\mathrm{d}}$

$$
V_{i}(\epsilon)=\left(\bar{Z}_{i}(\epsilon), 0, \epsilon \Phi_{s}^{*-1} X_{i}(\epsilon \cdot) U, 0\right)
$$

and

$$
V_{0}(\epsilon)=\left(0,0,0, \sum_{i>0}\left\langle U^{-1} \Phi_{s}^{*-1} X_{i}(\epsilon \cdot), \cdot\right\rangle^{2}\right)
$$

Let be the generator

$$
L_{t o t, \epsilon}\left(h_{x, y}\right)=1 / 2 \sum_{i>0} V_{i}^{2}(\epsilon)+\bar{Z}_{0}(\epsilon)+V_{0}(\epsilon)
$$

It generates a time inhomogeneous semi-group. We have

Lemma 5. For all positive $p$, the uniform Malliavin condition is checked:

$$
\sup _{\epsilon<1} \exp \left[L_{t o t, \epsilon}\left(h_{x, y}\right)\right]\left[V^{-p}\right](0,0,0, I, 0)<\infty
$$

Theorem 1 is a consequence of the next theorem, (which is an extension of Theorem 1 of [21]) and of (39):

Theorem 6. When $\epsilon \rightarrow 0, \quad r_{\epsilon}(0) \rightarrow r_{0}(0)$ where $r_{0}(\cdot)$ is the density of the measure which to fassociates

$$
\exp \left[\tilde{R}_{0}\left(h_{x, y}\right)\right]\left[\exp \left[\left\langle p_{1}(x, y), u_{2}\right\rangle\right] f\right](0,0,0,0)
$$

First of all, we recall the Wentzel-Freidlin estimates translated in semi-group theory by Léandre [22,23,25]:

Theorem 7. (Wentzel-Freidlin) Let $Y_{i}$ some time dependent vector fields with bounded derivatives at each order on $\mathbb{R}^{\mathrm{d}_{1}}, i=0, \cdots, m$. We consider the control distance $\mathrm{d}^{Y}\left(x_{1}, y_{1}\right)$ as in (8) and the diffusion semi-group $\exp \left[1 / 2 \epsilon^{2} \sum_{i>0} Y_{i}^{2}+\epsilon^{2} Y_{0}\right]$. We suppose that the control distance is continuous. Then for any open subset $O$

$$
\begin{aligned}
& \varlimsup_{\epsilon \rightarrow 0} 2 \epsilon^{2} \log \left(\exp \left[1 / 2 \epsilon^{2} \sum Y_{i}^{2}+\in Y_{0}\right]\left[1_{O}\right]\left(x_{1}\right)\right) \\
& \leq-\inf _{y_{1}^{\prime} \in O} \mathrm{~d}_{Y}^{2}\left(x_{1}, y_{1}^{\prime}\right)
\end{aligned}
$$

Proof of Theorem 6. Let $\chi$ be a smooth function from $\mathbb{R}$ into $[0,1]$ equals to 1 and 0 and equals to 0 if $|v|>\rho$. By Wentzel-Freidlin estimates, we can find an $\eta>0$ such that if $p>1$. 


$$
\exp \left[\tilde{R}_{x, y}\right]\left[\exp \left[p<p_{1}(x, y), \Phi_{1}\left(\epsilon x^{\prime}\right)-y-\epsilon u_{1}>/ \epsilon^{2}\right] \chi\left(x^{\prime}\right)(1-\chi)(v)\right](0,0,0,0) \leq \exp \left[-\eta \epsilon^{-2}\right]
$$

By the integration by part of the Malliavin Calculus and the Technical Lemma 5, we have if $\alpha$ is a multi-index

$$
\left|\exp \left[\tilde{R}_{x, y}\right]\left[\exp \left[\left\langle p_{1}(x, y), \Phi_{1}\left(\epsilon x^{\prime}\right)-y-\epsilon u_{1}\right\rangle / \epsilon^{2}\right] \chi\left(x^{\prime}\right)(1-\chi)(v) D^{(\alpha)} f\right](0,0,0,0)\right| \leq\|f\|_{\infty} \exp \left[-\eta / \epsilon^{2}\right]
$$

Therefore we have only to estimate the density in 0 of the measure which to $f$ associate

$$
\exp \left[\tilde{R}_{\epsilon}\left(h_{x, y}\right)\right]\left[\exp \left[\left\langle p_{1}(x, y), \Phi_{1}\left(\epsilon x^{\prime}\right)-y-\epsilon u_{1}-\epsilon^{2} / 2 u_{2}\right\rangle / \epsilon^{2}\right] \exp \left[\left\langle p_{1}(x, y), u_{2}\right\rangle / 2\right] \chi\left(x^{\prime}\right) \chi(v) f\right][0,0,0,0]
$$

By using Lemma 3, Lemma 4, Lemma 5 the density of this measure tends to $r_{0}(0)$ by using the Malliavin Calculus of Bismut type which depends of a parameter of [21].

\section{Proof of the Technical Lemmas}

Proof of Lemma 3. Let us first show that

$$
\exp \left[\tilde{R}_{\epsilon}\left(h_{x, y}\right)\right]\left[\left|\Phi_{1}\left(\epsilon x^{\prime}\right)-y-\epsilon u_{1}-\epsilon^{2} / 2 u_{2}\right|^{2} / \epsilon^{4}\right] \rightarrow 0
$$

(We will omitt to write later the obvious initial condition which appear in various semi-group later). We introduce a polynomial $F$ of degre less or equal to 2 in $\Phi_{1}\left(\epsilon x^{\prime}\right)$ and in $u_{1}, u_{2}$. Let us compute the Taylor expansion of $\exp \left[\tilde{R}_{\epsilon}\left(h_{x, y}\right)\right][F]$. We use Lemma 1 of [21]. If the degree of $F$ in $\Phi_{1}\left(\epsilon x^{\prime}\right)$ is 2 , the two first terms of the Taylor expansion are 0 and the term of order 2 is

$$
\exp \left[\tilde{R}_{0}\left(h_{x, y}\right)\right]\left[D^{2} F\left(0, u_{1}, u_{2}\right) u_{1} \cdot u_{1}\right]
$$

where we take partial derivatives in the first component. If the polynomial $F$ is of degree 1 in $\Phi_{1}\left(\epsilon x^{\prime}\right)$, the term of order 1 is

$$
\exp \left[\tilde{R}_{0}\left(h_{x, y}\right)\right]\left[D F\left(0, u_{1}, u_{2}\right) u_{1}\right]
$$

and the term of order two is

$$
\exp \left[\tilde{R}_{0}\left(h_{x, y}\right)\right]\left[D F\left(0, u_{1}, u_{2}\right) u_{2}\right]
$$

Lemma 3 will arise from the translation in semi-group theory of Lemma 3.4 of [12].

For all $p$ there exists a $\rho$ such that

$$
\begin{aligned}
& \sup _{\epsilon<1} \exp \left[\tilde{R}_{\epsilon}\left(h_{x, y}\right)\right] \\
& {\left[\exp \left[p\left\langle\Phi_{1}\left(\epsilon x^{\prime}\right)-\epsilon u_{1}-\epsilon^{2} / 2 u_{2}, p_{1}(x, y)\right\rangle\right] ; 1_{|v| \leq \rho}\right]<\infty}
\end{aligned}
$$

The proof follows slightly the line of Lemma 3.4 of [12]. We don't write the convenient enlarged semigroups when we enlarge the space. We follow the notation of [12], $\eta$ being replaced by $\epsilon$ and $V_{\alpha}$ being replaced by $X_{i}$.
We introduce the new coordinate

$$
\eta_{s}^{\epsilon}=1 / \epsilon\left(\Phi_{s}\left(\epsilon x^{\prime}\right)-x_{s}\left(h_{x, y}\right)\right)
$$

We use the Itô formula in semi-group theory of [25]. This leads to introduce extra coordinates in the vector fields:

1) $X_{i}\left(\Phi_{s}\left(\epsilon x^{\prime}\right)\right)$
2) $\partial X_{i}(s) \eta_{s}^{\epsilon} \mathrm{d} / \mathrm{d} s h_{x, y, s}^{i}$

$=\int_{0}^{1} D\left(x_{s}\left(h_{x, y}+u\left(\Phi_{s}\left(\epsilon x^{\prime}\right)-x_{s}\left(h_{x, y}\right)\right) \mathrm{d} u \eta_{s}^{\epsilon} \mathrm{d} / \mathrm{d} s h_{x, y, s}^{i}\right.\right.$

We introduce the new variable $\Xi_{s}^{\varepsilon}$ which is associated to the extra component vector fields

3) $\sum \partial X_{i}(s) \Xi_{s}^{\epsilon} \mathrm{d} / \mathrm{d} s h_{x, y, s}^{i}$.

We use another time the Itô formula in semi-group theory of [25] (11). This leads to introduce the vector field associated to another variable $\eta_{s}^{1, \epsilon}$.

4) $\left(\Xi_{s}^{\epsilon}\right)^{-1} X_{i}\left(\Phi_{s}\left(\epsilon x^{\prime}\right)\right)$

We introduce an extra variable $\eta_{s}^{3, \epsilon}$ associated to another component in the drift which is $\left(\eta_{s}^{\epsilon}\right)^{2}$.

We get for another enlarged semi-group $\exp \left[\hat{R}_{\epsilon}^{1}\left(h_{x, y}\right)\right]$ an extension of formula 3.44 of [12], but with $\int_{0}^{1}\left|\eta_{s}^{\epsilon}\right|^{2} \mathrm{~d} s$ instead of $\sup \left|\eta_{s}^{\epsilon}\right|^{2}$.

Lemma 8. For all $\rho$, there exists $p_{0}>0$ such that

$$
\sup _{\epsilon<1} \exp \left[\hat{R}_{\epsilon}^{1}\left(h_{x, y}\right)\right]\left[\exp \left[p_{0} \eta_{1}^{3, \epsilon}\right] 1_{|v| \leq \rho}\right]<\infty
$$

We postpone later the proof of this lemma which is an analog of the quasi-continuity lemma of [25].

Next we consider another enlarged semi-group to look the couple $\eta_{s}^{\epsilon}$ and $\eta_{s}$ together. We use the Itô formula in semi-group theory of [25] (11), [22,23]. We introduce

1) $\partial^{2} X_{i}^{\epsilon}(s)$

$=2 \int_{0}^{1} \mathrm{~d} u \int_{0}^{u} D^{2} X_{i}\left(x_{s}\left(h_{x, y}\right)+v\left(\Phi_{s}\left(\epsilon x^{\prime}\right)-x_{s}\left(h_{x, y}\right)\right)\right) \mathrm{d} v$.

By introducing a cascade of vector fields, we can translate in semi-group theory (3.45) of [12]. We introduce a variable $\eta_{s}^{4, \varepsilon}$ associate to the new component in 
the drift $\left|\eta_{s}^{\epsilon}-\eta_{s}\right|^{2}$ and we can state an analog of Lemma 8 for a convenient enlarged semi-group $\exp \left[\hat{R}_{\epsilon}^{2}\left(h_{x, y}\right)\right]$.

For every $p$, there exists a small $\rho$ such that

$$
\sup _{\epsilon \leq 1} \exp \left[\hat{R}_{\epsilon}^{2}\left(h_{x, y}\right)\right]\left[\exp \left[p \eta_{1}^{4, \epsilon}\right] 1_{|v| \leq \rho}\right]<\infty
$$

which is the analog of (3.46) in [12] where we have replaced $\sup _{s \leq 1}\left|\eta_{s}^{\epsilon}-\eta_{s}\right|^{2}$ by $\int_{0}^{1}\left|\eta_{s}^{\epsilon}-\eta_{s}\right|^{2} \mathrm{~d} s$.

Let be $\theta_{s}^{\epsilon}=\eta_{s}^{\epsilon}-\eta_{s} \epsilon$ and $\theta$ associated to the extracomponent vector fields:

1) $\partial V_{i}\left(x_{s}\left(h_{x, y}\right)\right) \eta_{s}$ for the diffusion part.

2) $\sum \partial X_{i}\left(x_{s}\left(h_{x, y}\right) \eta_{s} \mathrm{~d} / \mathrm{d} s h_{s, x, y}^{i}\right.$

$+1 / 2 \sum \partial^{2} X_{i}\left(x_{s}\left(h_{x, y} \eta_{s}\right) \otimes \eta_{s}\right.$ for the drift part.

We use another time the Itô formula in semi-group theory of [25] (11) for a convenient enlarged semi-group established to study together $\theta_{s}^{\epsilon}$ and $\theta_{s}$. This allow to study $\theta_{s}^{\epsilon}-\theta$ and we conclude exactly as in pages 29 , 30 of [12] with a small improvement of Lemma 8 to study (3.46), (3.47) of [12]. $\square$

Proof of Lemma 4. We assemble the semi-group $\exp \left[\tilde{R}_{\epsilon}\left(h_{x, y}\right)\right]$ and the semi-group $\exp \left[\tilde{R}_{0}\left(h_{x, y}\right)\right]$ together in a total semi-group $\exp \left[\tilde{R}_{\epsilon}^{\text {tot }}\left(h_{x, y}\right)\right]$. We have some variables $x_{\epsilon}^{\prime}, u_{1}, u_{2}$ and $v$. We have

$$
D \Phi(0) \cdot x_{0}^{\prime}=u_{1}
$$

Let $\rho_{1}$ be small and $\rho$ be very small. We use the exponential inequality in semi-group theory of Lemma 8 . For a small $\rho$ and a small $\rho_{1}$, we have (we omitt to write the obvious initial values in the considered semigroups)

$$
\begin{aligned}
& \exp \left[\tilde{R}_{\epsilon}^{\text {tot }} h_{x, y}\right]\left[\left\langle u_{2}, p_{1}(x, y)\right\rangle>\eta^{-2} ;\left|x_{\epsilon}^{\prime}\right|<\rho ;|v|<\rho\right] \\
\leq & \exp \left[\tilde{R}_{\epsilon}^{\text {tot }} h_{x, y}\right] \\
& {\left[\left\langle u_{2}, p_{1}(x, y)\right\rangle>\eta^{-2} ; C \eta\left|D \Phi(0) x_{\epsilon}^{\prime}-u_{1}\right|>\rho_{1} ;|v|<\rho\right] } \\
+ & \exp \left[\tilde{R}_{\epsilon}^{\text {tot }} h_{x, y}\right]\left[\left\langle u_{2}, p_{1}(x, y)\right\rangle>\eta^{-2} ; \eta\left|u_{1}\right|<\rho_{1}\right] \\
= & A_{1}+A_{2}
\end{aligned}
$$

We choose a small $\rho_{1}$ and a very small $\rho$. The exponential inequalities of the proof of Lemma 8 show

$$
A_{1} \leq \exp \left[-C \eta^{-2}\right]
$$

It remains to estimate $A_{2}$. We scale the vector fields $Y_{1}(1)$ by $\eta Y_{i}(1)$ and $Y_{i}(2)$ by $\eta Y_{i}(2)$. We get a generator $\bar{R}_{\eta}\left(h_{x, y}\right)$ and a new Markov semi-group $\exp \left[u \bar{R}_{\eta}\left(h_{x, y}\right)\right]$. By a scaling argument, we recognize in

$$
A_{1} \quad \exp \left[\bar{R}_{\eta}\left(h_{x, y}\right)\right]\left[\left\langle u_{2}, p_{1}(x, y)\right\rangle>1,\left|u_{1}\right| \leq \rho_{1}\right](0,0,0)
$$

By a simple improvement of the large deviation estimates of Theorem 7, we get

$$
\begin{aligned}
& \varlimsup_{\eta \rightarrow 0} \log \exp \left[\bar{R}_{\eta}\left(h_{x, y}\right)\right] \\
& \cdot\left[\left\langle u_{2}, p_{1}(x, y)\right\rangle>1,\left|u_{1}\right| \leq \rho_{1}\right](0,0,0) \\
& =-\operatorname{minf}_{\left.\mid\left\langle x_{1}\left(h_{x, y}\right), k\right\rangle\right) \mid \leq \rho_{1} ;\left\langle p_{1}(x, y), D^{2} x_{1}\left(h_{x, y}\right) \cdot k \cdot k\right)>1}\|k\|^{2}
\end{aligned}
$$

We chose a small $\rho_{1}$ and we use (20) and the fact $(x, y)$ don't belong to the cut-locus in part 2 . We deduce that if $\rho$ is very small, that there exists a $C>1$ such that

$$
\begin{aligned}
& \exp \left[\tilde{R}_{\epsilon}\left(h_{x, y}\right)\right]\left[\left\langle u_{2}, p_{1}(x, y)\right\rangle / 2>\eta^{-2},\left|x^{\prime}\right|<\rho ;|v|<\rho\right] \\
\leq & \exp \left[-C \eta^{-2}\right]
\end{aligned}
$$

Remark. This result is traditionnally hold by using the theory of Fredholm determinant.

Proof of Lemma 5. We assemble together the semigroup $L_{t o t, \epsilon}\left(h_{x, y}\right)$ and $L_{t o t, 0}\left(h_{x, y}\right)$ in a global generator $\bar{L}_{t o t, \epsilon}\left(h_{x, y}\right)$ We get therefore a total semi-group $\exp \left[u \bar{L}_{t o t, \epsilon}\left(h_{x, y}\right)\right]$. We get the Malliavin matrix $V_{\varepsilon}$ and $V_{0}$. But $V_{0}$ is nothing else that $\left\langle U_{1}^{-1} D x_{1}\left(h_{x, y}\right), U_{1}^{-1} D x_{1}\left(h_{x, y}\right)\right\rangle$ which is invertible because $(x, y)$ don't belong to the cut-locus of the subriemannian geometry.

Moreover, by omitting to write the obvious starting conditions, we get for a small $\eta$ :

$$
\exp \left[\bar{L}_{t o t, \epsilon}\left(h_{x, y}\right)\right]\left[\left|V_{\epsilon} V_{0}^{-1}\right|>\eta\right] \leq C \epsilon^{p}
$$

for all $p$. Therefore for a small $\eta$ :

$$
\begin{aligned}
& \exp \left[\bar{L}_{t o t, \epsilon}\left(h_{x, y}\right)\right]\left[V_{\epsilon}^{-p}\right] \leq A_{1}+A_{2} \\
= & \exp \left[\bar{L}_{t o t, \epsilon}\left(h_{x, y}\right)\right]\left[V_{\epsilon}^{-p} ; 1_{\mid V_{\epsilon} V_{0}^{-1}>>\eta}\right] \\
+ & \exp \left[\bar{L}_{t o t, \epsilon}\left(h_{x, y}\right)\right]\left[\left(V_{\epsilon}-V_{0}+V_{0}\right)^{-p} ; 1_{\left|V_{\epsilon} V_{0}^{-1}\right|>\eta}\right]
\end{aligned}
$$

Since $V_{0}$ is constant invertible, $A_{2}$ is bounded independent of $p$ if $\eta$ is small enough. By the results of [22,23], there exist $n(p)$ such that:

$$
\exp \left[\bar{L}_{t o t, \epsilon}\left(h_{x, y}\right)\right]\left[V_{\epsilon}^{-p}\right] \leq C \epsilon^{-n(p)}
$$

By Hoelder inequality, we deduce that $A_{1}$ is bounded 
independent of $p$.

Proof of Lemma 8. This follows clearly the line of the quasi-continuity lemma for Wentzel-Freidlin estimates in semi-group theory of [25]. We sketch the proof.

We recall the elementary Kolmogorov lemma of the theory of stochastic processes ([26,27]).

Let $s \rightarrow X_{s}$ be a family of random variables parametrized by $s \in[0,1]$ with values in $\mathbb{R}^{\mathrm{d}}$ equals to 0 or 1 in $s=0$ such that

$$
E\left[\left|X_{s^{\prime}}-X_{s}\right|^{p}\right] \leq C(p)\left(s^{\prime}-s\right)^{\alpha p}
$$

for $s^{\prime}>s$. There exists a continuous version of $s \rightarrow X_{s}$ and the $L^{p}$ norm of $\left(X_{1}\right)^{*}=\sup _{s \leq 1}\left|X_{s}\right|$ can be estimated only in terms of the data (73).

Let us recall that $R_{\eta}^{1}\left(h_{x, y}\right)$ is a time dependent generator. For $s^{\prime}>s$ there is a time inhomogeneous semigroup $\exp \left[\left(R_{\epsilon}^{1}\right)_{s}^{s^{\prime}}\left(h_{x, y}\right)\right]$. By the Burkholder-DaviesGundy inequality in semi-group theory of [16] (19), we have

$$
\exp \left[\left(R_{\epsilon}^{1}\right)_{0}^{s}\right]\left[\exp \left[\left(R_{\epsilon}^{1}\right)_{s}^{s^{\prime}}\right]\left[\left|\eta_{s}^{1, \epsilon}-\eta_{s^{\prime}}^{1, \epsilon p}\right|\right] \leq C(p) t^{\alpha p}\right.
$$

There we can define a continuous stochastic process with probability measure $\mathrm{d} P$ associated to $\eta_{s}^{1, \epsilon}$.

We use the Paul Levy martingale exponential in semigroup theory of [25] (33), (46). We get

$$
\begin{aligned}
& E_{P}\left[\left|\exp \left[\left\langle A, \eta_{s^{\prime}}^{1, \epsilon}\right\rangle\right]-\exp \left[\left\langle A, \eta_{s^{\prime}}^{1, \epsilon}\right\rangle\right]\right|^{p}\right] \\
\leq & C(p)\left(s^{\prime}-s\right)^{\alpha p} \exp \left[C|A|^{2}\right]
\end{aligned}
$$

By the Kolmogorov lemma, we get

$$
E_{P}\left[\left(\exp \left\langle A, \eta_{1}^{1, \epsilon}\right\rangle\right)^{*}\right] \leq C \exp \left[C|A|^{2}\right]
$$

By standard computations, we deduce that

$$
P\left[\left(\eta_{1}^{1, \epsilon}\right)^{*}>C\right] \leq K^{\prime} \exp \left[-K C^{2}\right]
$$

But $\left(\Xi_{s}^{\varepsilon}\right)$ is bounded, and by the same type of argument we deduce that

$$
P\left[\left(\eta_{1}^{\epsilon}\right)^{*}>C\right] \leq K^{\prime} \exp \left[-K C^{2}\right]
$$

But

$$
\eta_{1}^{3, \epsilon}=\int_{0}^{1}\left|\eta_{s}^{\epsilon}\right|^{2} \mathrm{~d} s
$$

such that

$$
\sup _{\epsilon \leq 1} \exp \left[\hat{R}_{\epsilon}^{1}\left(h_{x, y}\right)\right]\left[\eta_{1}^{3, \epsilon}>C\right] \leq K^{\prime} \exp \left[-K C^{2}\right]
$$

\section{Conclusion}

We have translated in semi-group theory some classical result of stochastic analysis for subelliptic heat-kernels where Bismutian non degeneracy condition [7] plays a preominent role.

\section{REFERENCES}

[1] L. Hoermander, "Hypoelliptic Second Order Differential Equations," Acta Mathematica, Vol. 119, No. 1, 1967, pp. 147-171. doi:10.1007/BF02392081

[2] P. Malliavin, "Stochastic Calculus of Variations and Hypoelliptic Operators,” In: K. Itô, Ed., Stochastic Analysis, Kinokuniya, Tokyo, 1978, pp. 195-263.

[3] R. Léandre, "Malliavin Calculus of Bismut Type in SemiGroup Theory," Far East Journal of Mathematical Sciences, Vol. 30, 2008, pp. 1-26.

[4] R. Léandre, "Malliavin Calculus of Bismut Type without Probability," In: V. S. Sunder and A. M. Boutet de Monvel, Eds., Festchrift in Honour of K. Sinha, Proceedings of Indian Academy Sciences-Mathematical Sciences, Vol. 116, 2006, pp. 507-518.

[5] M. Gromov, "Carnot-Caratheodory Spaces Seen from within,” In: A. Bellaiche, Ed., Sub-Riemannian Geometry, Birkhauser, Boston, 1996, pp. 79-323. doi:10.1007/978-3-0348-9210-0_2

[6] I. Kupka, “Géométrie Sous-Riemannienne," In Séminaire Bourbaki, Astérisque, Vol. 241, 1997, pp. 351-380.

[7] J. M. Bismut, "Large Deviations and the Malliavin Calculus,” Birkhauser, Boston, 1984.

[8] R. Léandre, "Estimation en Temps Petit de la Densité d'Une Diffusion Hypoelliptique," C. R. A. S. Série I, Vol. 301, 1985, pp. 801-804.

[9] R. Léandre, "Intégration dans la Fibre Associée a une Diffusion Dégénérée," Probability Theory and Related Fields, Vol. 76, No. 3, 1987, pp. 341-358. doi:10.1007/BF01297490

[10] G. Ben Arous, "Méthode de Laplace et de la Phase Stationnaire sur 1'Espace de Wiener," Stochastic, Vol. 25, No. 3, 1988, pp. 125-153. doi:10.1080/17442508808833536

[11] S. Takanobu and S. Watanabe, "Asymptotic Expansion Formulas of Schilder Type for a Class of Conditional Wiener Functional Integration," In: K. D. Elworthy and N. Ikeda, Eds., Asymptotic Problems in Probability Theory: Wiener Functionals and Asymptotics, Longman, New York, 1992, pp. 194-241.

[12] S. Watanabe, "Analysis of Wiener Functionals (Malliavin Calculus) and Its Applications to Heat Kernels," Annals of Probability, Vol. 15, No. 1, 1987, pp. 1-39. doi:10.1214/aop/1176992255

[13] T. J. S. Taylor, "Off Diagonal Asymptotics of Hypoelliptic Diffusion Equations and Singular Riemannian Geometry," Pacific Journal of Mathematics, Vol. 136, No. 2, 1989, pp. 379-394. doi:10.2140/pjm.1989.136.379

[14] S. Kusuoka, "More Recent Theory of Malliavin Calculus," Sugaku Expositions, Vol. 5, 1992, pp. 155-173.

[15] R. Léandre, “Appliquations Quantitatives et Qualitatives du Calcul de Malliavin,” In: M. Métivier and S. Watanabe, 
Eds., Stochastic Analysis, L. N. M., Vol. 1322, Springer, Berlin, 1988, pp. 109-134.

[16] S. Watanabe, "Stochastic Analysis and Its Applications," Sugaku, Vol. 5, 1992, pp. 51-72.

[17] F. Baudoin, "An Introduction to the Geometry of Stochastic Flows," Imperial College Press, London, 2000.

[18] E. B. Davies, "Heat Kernels and Spectral Theory," Cambridge University Press, Cambridge, 1992.

[19] N. Varopoulos, L. Saloff-Coste and T. Coulhon, "Analysis and Geometry on Groups," Cambridge University Press, Cambridge, 1992.

[20] D. Jerison and A. Sanchez-Calle, "Subelliptic Differential Operators," In: C. Berenstein, Ed., Complex Analysis III, L. N. M., Vol. 1277, Springer, Berlin, 1987, pp. 46-77. doi:10.1007/BFb0078245

[21] R. Léandre, "Varadhan Estimates without Probability: Lower Bounds," In: D. Baleanu, et al., Eds., Mathematical Methods in Engineerings," Springer, Berlin, 2007, pp. 205-217.

[22] R. Léandre, "Varadhan Estimates in Semi-Group Theory:
Upper Bound," In: M. Garcia-Planas, et al., Eds., Applied Computing Conference, WSEAS Press, Athens, 2008, pp. 77-81.

[23] R. Léandre, "Large Deviations Estimates in Semi-Group Theory," In: T. E. Simos, et al., Eds., Numerical Analysis and Applied Mathematics, A. I. P. Proceedings, American Institute Physics, Melville, 2008, pp. 351-355.

[24] B. Gaveau, "Principe de Moindre Action, Propagation de la Chaleur et Estimées Sous-Elliptique sur Certains Groupes Nilpotents," Acta Mathematica, Vol. 107, 1977, pp. 43-101.

[25] R. Léandre, "Wentzel-Freidlin Estimates in Semi-Group Theory," In: Y. C. Soh, Ed., Control, Automation Robotics and Vision, 2008, pp. 2233-2235.

[26] P. A. Meyer, "Flot d'Une Équation Différentielle Stochastique," In: P. A. Meyer, et al., Eds., Séminaire de Probabilités $X V$, L. N. M., Vol. 850, Springer, Berlin, 1981, pp.100-117.

[27] P. Protter, "Stochastic Integration and Differential Equations," Springer, Berlin, 1995. 